\title{
Orchestrating the unfolded protein response in health and disease
}

\author{
Randal J. Kaufman \\ Department of Biological Chemistry, Howard Hughes Medical Institute, University of Michigan Medical Center, \\ Ann Arbor, Michigan, USA
}

J. Clin. Invest. 110:1389-1398 (2002). doi:10.1172/JCI200216886.

The endoplasmic reticulum (ER), the entrance site for proteins destined to reside in the secretory pathway or the extracellular environment, is also the site of biosynthesis for steroids and for cholesterol and many lipids. Given the considerable number of resident structural proteins and biosynthetic enzymes and the high expression of many secreted proteins, the total concentration of proteins in the this organelle can reach $100 \mathrm{mg} / \mathrm{ml}$. The ER relies on an efficient system of protein chaperones that prevent the accumulation of unfolded or aggregated proteins and correct misfolded proteins that are caught in low-energy kinetic traps (see Horwich, this Perspective series, ref. 1).

These chaperone-mediated processes expend metabolic energy to ensure high-fidelity protein folding in the lumen of the ER. For example, the most abundant ER chaperone, BiP/GRP78, uses the energy from ATP hydrolysis to promote folding and prevent aggregation of proteins within the ER. In addition, the oxidizing environment of the ER creates a constant demand for cellular protein disulfide isomerases to catalyze and monitor disulfide bond formation in a regulated and ordered manner. Operating in parallel with chaperonedependent protein folding are several "quality control" mechanisms, which ensure that, of all proteins translocated into the ER lumen, only those that are properly folded transit to the Golgi compartment. Proteins that are misfolded in the ER are retained until they reach their native conformation or are retrotranslocated back

\footnotetext{
Address correspondence to: Randal J. Kaufman, Department of Biological Chemistry, Howard Hughes Medical Institute, University of Michigan Medical Center, 1150 W. Medical Center Drive, Ann Arbor, Michigan 48109-0650, USA.

Phone: (734) 763-9037; Fax: (734) 763-9323;

E-mail: kaufmanr@umich.edu.

Conflict of interest: The author has declared that no conflict of interest exists.

Nonstandard abbreviations used: endoplasmic reticulum (ER); unfolded protein response (UPR); basic leucine zipper (bZIP); UPR element (UPRE); endoplasmic reticulum stress response element (ERSE); site-1 protease (S1P); site-2 protease (S2P); sterol-response element-binding protein (SREBP); eukaryotic translation initiation factor 2 (eIF2); endoplasmic reticulum-associated degradation (ERAD); $\alpha 1$-proteinase inhibitor ( $\alpha 1-\mathrm{PI})$; cystic fibrosis transmembrane conductance regulator (CFTR); autosomal recessive juvenile parkinsonism (AR-JP).
}

into the cytosol for degradation by the $26 \mathrm{~S}$ proteasome. The ER has evolved highly specific signaling pathways to ensure that its protein-folding capacity is not overwhelmed. These pathways, collectively termed the unfolded protein response (UPR), are required if the cell is to survive the ER stress (see Ron, this Perspective series, ref. 2) that can result from perturbation in calcium homeostasis or redox status, elevated secretory protein synthesis, expression of misfolded proteins, sugar/glucose deprivation, or altered glycosylation. Upon accumulation of unfolded proteins in the ER lumen, the UPR is activated, reducing the amount of new protein translocated into the ER lumen, increasing retrotranslocation and degradation of ER-localized proteins, and bolstering the protein-folding capacity of the ER. The UPR is orchestrated by the coordinate transcriptional activation of multiple genes, a general decrease in translation initiation, and a concomitant shift in the mRNAs that are translated.

The recent discovery of the mechanisms of ER stress signaling, coupled with the ability to genetically engineer model organisms, has led to major new insights into the diverse cellular and physiological processes that are regulated by the UPR. Here, I summarize current discoveries that have offered insights into the complex regulation of the UPR and its relevance to human physiology and disease.

\section{Glucose and protein folding}

Early studies demonstrated that both viral transformation and glucose depletion induce transcription of a set of related genes that were termed glucose-regulated proteins (GRPs) (3). Since viral transformation increases both the cellular metabolic rate and ATP utilization, it became evident that, in both cases, this signal emanates from the ER as a consequence of energy deprivation. Because proteins have different ATP requirements for protein folding prior to export, it has been proposed that the threshold for UPR activation might differ among various cell types, depending on their energy stores and the amount and nature of the secretory proteins they produce (4).

Glucose not only provides the metabolic energy needed by cells but also participates directly in glycoprotein folding as a component of oligosaccharide structures. 
The recognition and modification of oligosaccharide structures in the lumen of the ER is intimately coupled to polypeptide folding (5). As the growing nascent chain is translocated into the lumen of the ER, a 14-oligosaccharide core $\left(\mathrm{GlcNAc}_{2} \mathrm{Man}_{9} \mathrm{Glc}_{3}\right)$ is added to consensus asparagine residues. Immediately after the addition of this core, the three terminal glucose residues are cleaved by the sequential action of glucosidases I and II to yield a GlcNAc ${ }_{2} \mathrm{Man}_{9}$ structure. If the polypeptide is not folded properly, a UDP-glucose:glycoprotein glucosyltransferase (UGGT) recognizes the unfolded nature of the glycoprotein and reglucosylates the core structure to re-establish the glucose- $\alpha(1,3)$-mannose glycosidic linkage. Monoglucosylated oligosaccharides containing this bond bind to the ER-resident protein chaperones calnexin and calreticulin.

This quality control process ensures that unfolded glycoproteins do not exit the ER. Treatment of cells with castanospermine, a transition-state analogue inhibitor of glucosidases I and II, inhibits this monoglucosylation cycle, prevents interaction of unfolded glycoproteins with calnexin and calreticulin, and activates the UPR. Genetic alterations that reduce the nucleotide sugar precursor pool or glycosyltransferase reactions likewise activate the UPR (6). Therefore, the recognition of altered carbohydrate structures is in some manner linked to UPR activation.

\section{The UPR in yeast and higher eukaryotes}

On a cellular level, the accumulation of unfolded proteins in the ER lumen induces the transcription of a large set of genes whose products increase the ER's volume or its capacity for protein folding or promote the degradation of misfolded proteins through the process of ER-associated protein degradation (ERAD) (7). For example, transcription of the ER protein chaperone $\mathrm{BiP}$ is a classical marker for UPR activation in yeast and mammalian cells (8). BiP binds hydrophobic exposed patches on the surfaces of unfolded proteins and interactive sites on unassembled protein subunits, and it releases its polypeptide substrates upon ATP binding. In parallel, as Ron (this Perspective series, ref. 2) details in his accompanying article, translation is attenuated to decrease the protein-folding load. The complex network of physiological responses to ER stress is regulated by only a few ER transmembrane proteins: IRE1, PERK, and ATF6 (9). IRE1, PERK, and ATF6 are proximal sensors that regulate the production and/or quality of basic leucine zipper-containing (bZIP-containing) transcription factors that may form homo- and heterodimers. Combinatorial interactions of these factors generate diversity in responses for different subsets of UPRresponsive genes. In multicellular organisms, if these adaptive responses are not sufficient to relieve ER stress, the cell dies through apoptosis or necrosis.

\section{IRE1-dependent splicing}

The UPR-signaling pathway was first described less than ten years ago in the budding yeast Saccharomyces cerevisiae. Elegant studies identified IRE1 as the sensor of unfolded proteins in the ER lumen. IRE1 is a type 1 transmembrane Ser/Thr protein kinase that also has a site-specific endoribonuclease (RNase) activity. The presence of unfolded proteins in the ER lumen promotes dimerization and trans-autophosphorylation, rendering IRE1 active as an RNase, and allowing it to cleave a 252-base intron from the mRNA encoding the transcription factor HAC1 (10). The $5^{\prime}$ and $3^{\prime}$ ends of HAC1 mRNA are spliced together by tRNA ligase in a process that is independent of the spliceosome and the usual intranuclear machinery for mRNA splicing. Splicing of HAC1 mRNA increases its translational efficiency and alters sequence of the encoded HAC1 protein, yielding a potent transcriptional activator (11) that can bind and activate the UPR elements (UPREs) upstream of many UPR-inducible genes. In S. cerevisiae, the UPR activates transcription of approximately 381 genes (7).

All eukaryotic cells appear to have maintained the essential and unique properties of the UPR present in $S$. cerevisiae, but higher eukaryotes possess additional sensors that generate diverse, coordinately regulated responses that promote stress adaptation or cell death. The mammalian genome contains two homologues of yeast IRE1 - IRE $1 \alpha$ and IRE1 $\beta$. Whereas IRE $1 \alpha$ is expressed in most cells and tissues, with high-level expression in the pancreas and placenta (12), IRE1 $\beta$ expression is prominent only in intestinal epithelial cells (13). Both IRE1 molecules respond to the accumulation of unfolded proteins in the ER, which activate their kinase and, thereby, their RNase activities. The cleavage specificities of IRE $1 \alpha$ and IRE $1 \beta$ are similar, if not identical, suggesting that they do not recognize different sets of substrates but rather generate temporally specific and tissue-specific expression $(14,15)$.

Searching for transcription factors that mediate the UPR, Yoshida et al. defined a mammalian ER stress response element [ERSEI; CCAAT $\left(\mathrm{N}_{9}\right) \mathrm{CCACG}$ ] that is necessary and sufficient for UPR gene activation. Using a yeast one-hybrid screen, these authors isolated XBP1, a bZIP transcription factor X-box DNA binding protein (16). Subsequently, several groups demonstrated that XBP1 mRNA is a substrate for mammalian IRE1, much as the HAC1 mRNA in S. cerevisiae is processed by the yeast IRE1; this pathway is also conserved in Caenorhabditis elegans (17-20). On activation of the UPR, XBP1 mRNA is cleaved by IRE1 to remove a 26 -nucleotide intron and generate a translational frameshift. As expected given the precedent of HAC1 regulation in yeast, the resulting processed mRNA encodes a protein with a novel carboxy-terminus that acts as a potent transcriptional activator.

Overexpression of either IRE1 $\alpha$ or IRE1 $\beta$ is sufficient to activate transcription from a $B i P$ promoter reporter construct (15). Analysis of a minimal UPRE motif (TGACGTGC/A) (21) uncovered a transcriptional defect in IRE1 $\alpha$-null mouse embryo fibroblasts that could be complemented by expression of spliced XBP1 mRNA (20), and Yoshida et al. (unpublished data) recently identified a UPR-inducible gene that uniquely requires IRE1 $\alpha$-mediated splicing of XBP1 mRNA. However, neither IRE $1 \alpha$ nor IRE $1 \beta$ is necessary for transcriptional activation of the BiP gene, as judged by the phenotype of 
IRE1 $\alpha / \beta$-deleted murine cells $(20,22,23)$. These results indicate that a subset of UPR targets require IRE1 but that at least one IRE1-independent pathway exists for UPR-mediated transcriptional induction.

Deletion of IRE $1 \alpha$ causes embryonic lethality at embryonic day 10.5 (E10.5) (20, 22, 23). Therefore, although IRE1 $\alpha$ is not required for the UPR, it is clearly required for mammalian embryogenesis. XBP1 deletion also causes embryonic lethality, but the mutant embryos can survive up to day E14.5, consistent with the notion that XBP1 acts downstream of IRE1 $\alpha . X B P 1$ deletion causes cardiomyopathy and liver hypoplasia $(24,25)$. In contrast, IRE1 $\beta$-null mice develop normally but exhibit increased susceptibility to experimentally induced colitis, a phenotype that is consistent with the specific expression of this kinase in the intestinal epithelium (26).

\section{Activation of ATF6 and PERK by ER stress}

The activating transcription factor ATF6 (16) has been identified as another regulatory protein that, like XBP1, can bind ERSEI elements in the promoters of UPRresponsive genes. There are two forms of ATF6, both synthesized as ER transmembrane proteins. ATF $6 \alpha(90$ $\mathrm{kDa})$ and ATF6 $\beta$ (110 kDa, also known as CREB-RP) both require the presence of the transcription factor CBF (also called NF-Y) to bind ERSEI (27-30).

On activation of the UPR, both forms of ATF6 are processed to generate 50- to $60-\mathrm{kDa}$ cytosolic, bZIPcontaining transcription factors that migrate to the nucleus (27). Processing of ATF6 by site- 1 protease (S1P) and site- 2 protease (S2P) occurs within the transmembrane segment and at an adjacent site exposed to the ER lumen. S1P and S2P are the processing enzymes that cleave the ER-associated transmembrane sterolresponse element-binding protein (SREBP) upon cholesterol deprivation (31). The cytosolic fragment of cleaved SREBP migrates to the nucleus to activate transcription of genes required for sterol biosynthesis. Interestingly, although the mechanism regulating ATF6 processing is similar to that regulating SREBP processing (32), the UPR only elicits ATF6 processing, whereas sterol deprivation alone induces SREBP processing. The SREBP cleavage-activating protein (SCAP) confers specificity for SREBP transport to the Golgi compartment, and consequently cleavage in response to sterol deprivation (33). It is unknown whether another cleavage-activating protein, analogous to SCAP but active only following induction of the UPR, promotes the specific cleavage and activation of ATF6 by S1P and S2P.

Transcription of UPR-responsive genes is induced when the cleaved form of ATF 6 activates the XBP1 promoter. Therefore, signaling through ATF6 and IRE1 merges to induce XBP1 transcription and mRNA splicing, respectively (Figure 1, a and b). ATF6 increases XBP1 transcription to produce more substrate for IRE1mediated splicing that generates more active XBP1, providing a positive feedback for UPR activation. However, cells that lack either IRE1 $\alpha$ or ATF6 cleavage can induce XBP1 mRNA (20). These two pathways may thus pro- vide parallel signaling pathways for XBP1 transcriptional induction. Alternatively, another pathway - possibly mediated by the ER-localized protein kinase PERK (see Ron, this Perspective series, ref. 2) - may also contribute to induction of XBP1 mRNA. The binding specificities of XBP1 and ATF6 are similar, although ATF6 binding requires $\mathrm{CBF}$ binding to an adjacent site, whereas XBP1 binds independently $(17,20,21,34)$. These binding specificities provide another avenue for complementary interaction between the IRE1-XBP1 and ATF6 pathways at the level of transcriptional activation. In addition, these transcription factors might regulate transcription from a second ERSE (ERSEII), which also contains a CCACG motif (35).

In parallel with the activation of ATF6 processing and the consequent changes in gene transcription, the accumulation of unfolded proteins in the ER also alters cellular patterns of translation. The protein kinase PERK has been implicated in this aspect of the ER stress response (see Ron, this Perspective series, ref. 2). Activated PERK phosphorylates the $\alpha$ subunit of eukaryotic translation initiation factor 2 (eIF2 $\alpha$ ) and attenuates general protein synthesis. Inactivation of the PERK-eIF2 $\alpha$ phosphorylation pathway decreases cells' ability to survive ER stress $(36,37)$. The PERK pathway promotes cell survival not only by limiting the protein-folding load on the ER, but also by inducing transcription of UPR-activated genes, one-third of which require phosphorylation of eIF $2 \alpha$ for their induction (36). Preferential translation of the transcription factor ATF4 allows for continued activation of these genes under conditions of stress, when general protein synthesis is inhibited $(36,37)$.

\section{A coordinated mechanism for activation}

One puzzling question about the UPR is how three independent sensors are activated by a common stimulus, the accumulation of unfolded proteins in the ER lumen. BiP, which negatively regulates the UPR, interacts with all three sensors, IRE1, PERK, and ATF6, under nonstressed conditions and may indeed be the master regulator of UPR activation.

Upon accumulation of unfolded proteins in the ER, BiP is released from IRE1, PERK, and ATF6. It is believed that the unfolded proteins bind $\mathrm{BiP}$ and sequester it from interacting with IRE1, PERK, and ATF6 to elicit their activation. In this manner, BiP senses both the level of unfolded proteins and the energy (ATP) level in the cell in regulating the UPR. Following release from $\mathrm{BiP}$, IRE1 and PERK are each free to undergo spontaneous homodimerization mediated by their lumenal domains and to become phosphorylated by their endogenous kinase activities $(38,39)$. BiP interaction with ATF6 prevents trafficking of ATF 6 to the Golgi compartment. For this reason, BiP release permits ATF6 transport to the Golgi compartment, where it gains access to S1P and S2P proteases (32). The regulation of signaling through the free level of $\mathrm{BiP}$ is an attractive hypothesis providing a direct mechanism by which all three ER stress sensors could be activated by the same stimulus. In addition, the increase in BiP during the UPR would provide a negative feedback to turn off UPR signaling. However, in certain 

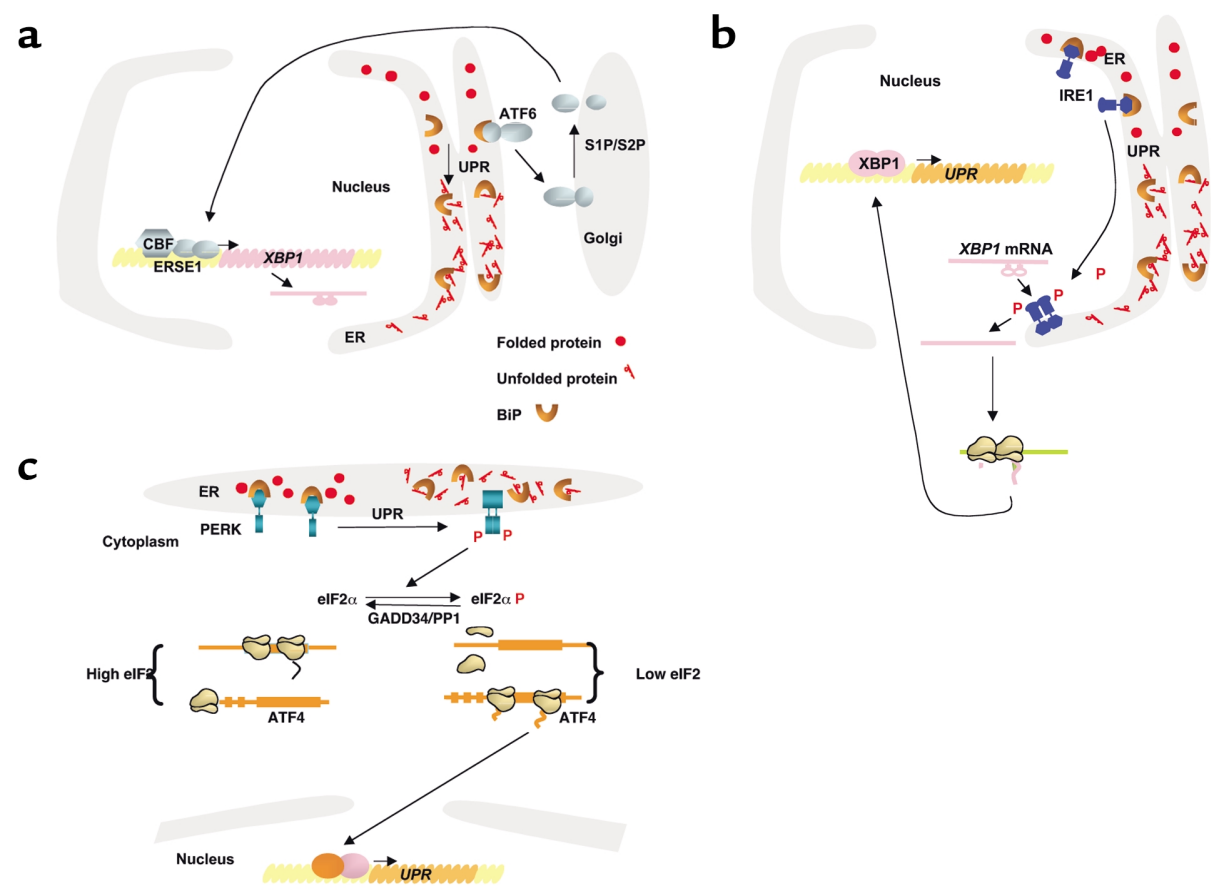

\begin{abstract}
Figure 1
Signaling the UPR in eukaryotes. Three proximal sensors, IRE1, PERK, and ATF6, coordinately regulate the UPR through their various signaling pathways. Whereas IRE1 and PERK are dispensable for many aspects of the response, ATF6 cleavage is required for UPR transcriptional induction and appears to be the most significant of these effectors in mammalian cells. BiP negatively regulates these pathways. BiP interacts with ATF6 to prevent its transport to the Golgi compartment (a). BiP binds to the lumenal domains of IRE1 (b) and PERK (c) to prevent their dimerization. As unfolded proteins accumulate, they bind BiP and reduce the amount of BiP available to bind and inhibit activation of IRE1, PERK, and ATF6. (a) BiP release from ATF6 permits transport to the Golgi compartment. In the Golgi, ATF6 is cleaved by S1P and S2P proteases to yield a cytosolic fragment that migrates to the nucleus to activate transcription of responsive genes, including XBP1. (b) BiP release from IRE1 permits dimerization to activate its kinase and RNase activities to initiate XBP1 mRNA splicing. XBP1 splicing removes a 26-base intron, creating a translational frameshift to yield a more potent transcriptional activator. (c) BiP release permits PERK dimerization and activation to phosphorylate Ser51 on elF2 $\alpha$ to reduce the frequency of AUG initiation codon recognition. As elF $2 \alpha$ phosphorylation reduces the functional level of elF2, the general rate of translation initiation is reduced. However, selective mRNAs, such as ATF4 mRNA, are preferentially translated under these conditions, possibly by the presence of open reading frames within the $5^{\prime}$ untranslated region of the mRNA. Upon recovery from the UPR, GADD34 targets PP1 to dephosphorylate elF2 $\alpha$ and increase protein translation.
\end{abstract}

cells, different stress conditions can selectively activate only one or two of the ER stress sensors. For example, in pancreatic $\beta$ cells, glucose limitation appears to activate PERK prior to activation of IRE1 (D. Scheuner and R.J. Kaufman, unpublished results). It will be important to elucidate how general BiP repression permits the selective activation of individual components of the UPR that mediate various downstream effects.

\section{The UPR as a mediator of programmed cell death} In contrast to UPR-signaling adaptation in response to ER stress, prolonged UPR activation leads to apoptotic cell death (Figure 2). The roles of several death-promoting signaling pathways have been shown by analysis of specific gene-deleted cells. Activated IRE1 recruits c-Jun-N-terminal inhibitory kinase (JIK) and the cytosolic adaptor TRAF2 to the ER membrane $(22,40)$. TRAF2 activates the apoptosis-signaling kinase 1 (ASK1), a mitogen-activated protein kinase kinase kinase (MAPKKK) (41). Activated ASK1 leads to activation of the JNK protein kinase and mitochondriadependent caspase activation (40-42).

ER insults lead to caspase activation by mitochondria/APAF-1-dependent and -independent pathways.
ER stress promotes cytochrome $c$ release from mitochondria, possibly by c-ABL kinase (43) or calcium (44). However, APAF1- $1^{--}$cells are susceptible to ER stress-induced apoptosis, indicating that the mitochondrial pathway is not essential (45). Caspase-12 is an ER-associated proximal effector in the caspase activation cascade, and cells lacking this enzyme are partially resistant to inducers of ER stress (46). ER stress induces TRAF2 release from procaspase 12 , allowing it to bind activated IRE1. As shown in Figure 2, release of TRAF2 permits clustering of procaspase- 12 at the ER membrane, leading to its activation (40). Caspase- 12 can activate caspase- 9 , which in turn activates caspase3 (47). Procaspase- 12 can also be activated by m-calpain in response to calcium release from the ER, although the physiological significance of this pathway is not known (48). In addition, upon ER stress, procaspase-7 is activated and recruited to the ER membrane (49). These findings support the notion that ER stress leads to several redundant pathways for caspase activation.

A second death-signaling pathway activated by ER stress is mediated by transcriptional activation of genes encoding proapoptotic functions. Activation of UPR sensor IRE1, PERK, or ATF6 leads to transcriptional 
activation of CHOP/GADD153, a bZIP transcription factor that potentiates apoptosis (see Ron, this Perspective series, ref. 2).

\section{The UPR in health and disease}

Primary amino acid sequence contains all the information for a protein to attain its final folded conformation. However, many folding intermediates exist along the folding pathway (see Horwich, this Perspective series, ref. 1), and some of these intermediates can become irreversibly trapped in low-energy states and activate the UPR. Clearance of such misfolded species requires a functional ER-associated degradation (ERAD) pathway, which is regulated by the UPR. Proteasomal degradation of ER-associated misfolded proteins is required to protect from UPR activation. Proteasomal inhibition is sufficient to activate the UPR, and, in turn, genes encoding several components of ERAD are transcriptionally induced by the UPR (7). Therefore, it is to be expected that UPR activation and impaired ERAD function might contribute to a variety of diseases and that polymorphisms affecting the UPR and ERAD responses could modify disease progression. The following examples provide the best available evidence linking the UPR pathway to the natural history of human diseases and animal models of these diseases.

\section{The UPR and ERAD in genetic disease}

Many recessive inherited genetic diseases are due to lossof-function mutations that disturb productive folding and that produce proteins that are either not secreted or not functional. In other cases, protein-folding mutations can interfere with cellular processes, resulting in a gain of function and a dominant pattern of inheritance. In several instances, UPR activation by the accumulation of unfolded proteins in the ER is known to contribute to disease progression. The distinction between these two classes of genetic disease is important, because gain-of-function protein-misfolding mutations will be less amenable to treatment by gene therapy to deliver a wild-type copy of the mutant gene.

One well-characterized protein-folding defect results from a mutation that leads to type 1 diabetes. The Akita mouse has a gain-of-function Cys96Tyr mutation in the proinsulin 2 (Ins2) gene; this mutation disrupts proinsulin folding. The mutant protein is retained in the ER of the pancreatic $\beta$ cell and activates the UPR. Crucially, the progressive development of diabetes in this model is not solely due to the lack of insulin but is rather a consequence of the misfolded protein accumulation, UPR activation, and $\beta$ cell death. When bred into a Chop ${ }^{-/-}$background, the Akita mutation causes a lesser degree of $\beta$ cell death and delayed onset of diabetes (50), indicating that the loss of at least

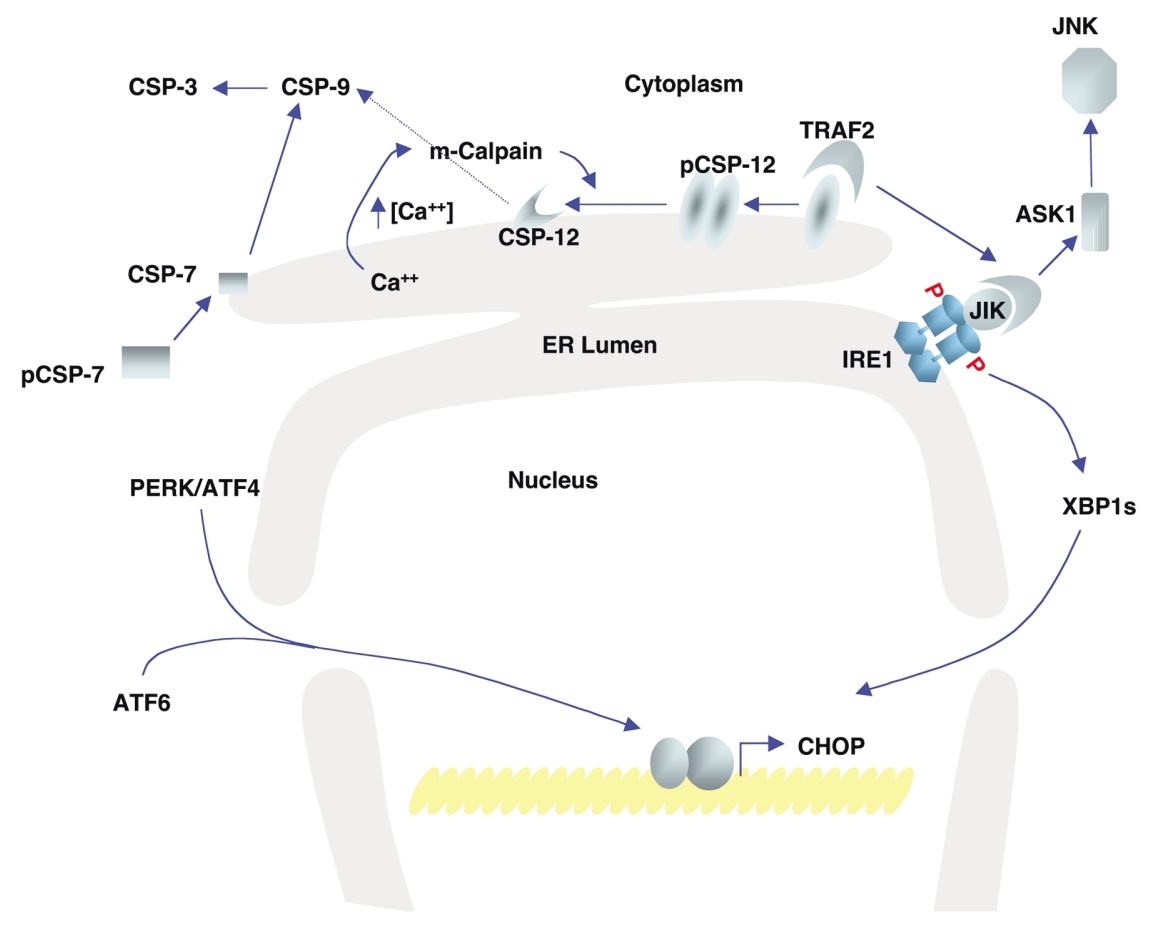

\section{Figure 2}

Signaling UPR-mediated cell death. The activation of procaspase-12 is likely the major pathway that induces apoptosis in response to ER stress. Upon activation of the UPR, c-Jun-N-terminal inhibitory kinase (JIK) release from procaspase-12 permits clustering and activation of procaspase-12. Caspase-12 activates procaspase-9 to activate procaspase-3, the executioner of cell death. In addition, activated IRE1 binds JIK and recruits TRAF2, which signals through apoptosis-signaling kinase 1 (ASK1) and JNK to promote mitochondria-dependent apoptosis. In addition, in vitro studies suggest that localized calcium release from the ER activates $\mathrm{m}$-calpain to cleave and activate procaspase-12. Upon UPR activation, procaspase-7 is activated and recruited to the ER membrane. Finally, IRE1, PERK, and ATF6 induce transcription of several genes encoding apoptotic functions, including $\mathrm{CHOP/GADD153.} \mathrm{CSP,} \mathrm{caspase;} \mathrm{pCSP,} \mathrm{procaspase.}$ 
one downstream signaling component of the UPR can ameliorate pathogenesis in this setting.

Deficiency in $\alpha 1$-proteinase inhibitor ( $\alpha 1-\mathrm{PI}$, also known as $\alpha 1$-antitrypsin) results in emphysema and destructive lung disease in one out of 1,800 births. However, a subgroup of affected individuals develop chronic liver disease and hepatocellular carcinoma as a consequence of a secretion defect in the misfolded protein at the site of synthesis, the hepatocyte. This is the most common genetic cause of liver disease in children. The $\mathrm{Z}$ allele of the $\alpha 1$ gene PI (Glu342Lys mutation) produces a protein that polymerizes and is retained in the ER for degradation by the proteasome (see Lomas and Mahadeva, this Perspective series, ref. 51; and Perlmutter, this series, ref. 52). While $\alpha 1$-PI $Z$ neither binds BiP nor activates the UPR, analysis of fibroblasts obtained from these patients demonstrates that individuals susceptible to liver disease have inherited a second trait that slows degradation of the misfolded protein in the ER (53), consistent with the idea that polymorphisms that reduce ERAD function can exacerbate pathogenesis of certain diseases.

There are numerous additional genetic misfolding diseases that are also likely influenced by UPR signaling. Because BiP release from IRE1, PERK, or ATF6 can activate the UPR, the expression of any wild-type or mutant protein that binds $\mathrm{BiP}$ can have a similar effect. In contrast, misfolded proteins that do not bind BiP are unlikely to activate the UPR. For example, cystic fibrosis is due to mutations in the cystic fibrosis transmembrane conductance regulator (CFTR) protein. Approximately $70 \%$ of patients with this disease carry a common mutation, deletion of Phe508, that results in a molecule that is retained in the ER and eventually degraded by the proteosome (see Gelman and Kopito, this Perspective series, ref. 54). Although expression of $\Delta 508$ CFTR does not activate the UPR in cultured cells, the protein does interact with calnexin, as well as HSP70, and requires ERAD function for cell survival.

Osteogenesis imperfecta (OI) results from misfolding mutations in procollagen that produce molecules that bind $\mathrm{BiP}$ and activate the UPR (55). Interestingly, Wolcott-Rallison syndrome is due to inactivating mutations in the PERK gene. Affected individuals, as well as mice with deletions in Perk, display osteoporosis and deficient mineralization throughout the skeletal system (56, 57), the same defects that are observed in OI. Procollagen type I accumulates to high levels and mature collagen is not detected in bone and osteoblasts from PERKnull mice. Osteoblasts from PERK-null humans and mice display fragmented and distended ER that is filled with electron-dense material $(56,57)$. These observations suggest that procollagen type 1 uniquely requires PERK function to maintain its transport out of the ER, processing, and secretion In this case, PERK may be required to limit procollagen synthesis so that it does not saturate the ER protein-folding capacity.

\section{The UPR and ERAD in conformational diseases}

Diseases caused by expansion of polyglutamine repeats and neurodegenerative diseases, such as Alzheimer dis- ease and Parkinson disease, represent a large class of conformational diseases associated with accumulation of abnormal protein aggregates in and around affected neurons. Recent evidence indicates that the pathogenesis of these diseases is due to a defect in proteasomal function that results in UPR activation, leading to cell death. The protein aggregates in these diseases are localized to the nucleus or the cytoplasm and would not be predicted to disturb ER function directly. Nevertheless, they have been found in some cases to activate the UPR and to promote cell death. Analysis of the polyglutamine repeat associated with the spinocerebrocellular atrophy protein (SCA3) in Machado-Joseph disease suggests that cytoplasmic accumulation of the SCA3 aggregate can inhibit proteasome function, thereby interfering with ERAD to induce the UPR and elicit caspase- 12 activation $(41,58)$. These findings support the idea that the UPR can signal the accumulation of unfolded proteins in the cytosol via proteasomal inhibition and disruption of ERAD function.

Parkinson disease is the most common movement disorder, affecting about $1 \%$ of individuals 65 years of age or older. Autosomal recessive juvenile parkinsonism (AR-JP) results from defects in the Parkin gene (59), which encodes a ubiquitin protein ligase (E3) that functions with ubiquitin-conjugating enzyme $\mathrm{UbcH} 7$ or $\mathrm{UbcH} 8$ to tag proteins for degradation. Overexpression of Parkin suppresses cell death associated with ER stress (60). Inherited Parkinson disease is associated with the accumulation in the ER of dopaminergic neurons of PAEL-R, a putative transmembrane receptor protein that is detected in an insoluble form in the brains of AR-JP patients (61). The accumulation of PAEL-R results from defective Parkin that does not maintain the proteasome-degrading activity necessary to maintain ER function (62). Other, still-unidentified substrates of the Parkin E3 ligase may also be relevant to the pathogenesis of AR-JP.

\section{The UPR in diabetes}

The metabolism of glucose is tightly controlled at the levels of synthesis and utilization through hormonal regulation. The most dramatic phenotype in Wolcott-Rallison syndrome is pancreatic $\beta$ cell death with infancyonset diabetes (56). A similar defect is observed in PERK-null mice; this defect also correlated with increased apoptosis of $\beta$ cells $(57,63)$. In addition, mice with a homozygous Ser51Ala mutation at the PERK phosphorylation site in eIF $2 \alpha$ display an even greater $\beta$ cell loss that appears in utero (36). Therefore, translational control through PERK-mediated phosphorylation of eIF $2 \alpha$ is required to maintain $\beta$ cell survival (see Ron, this Perspective series, ref. 2 ). The more severe $\beta$ cell loss in mice harboring the Ser51Ala eIF2 $\alpha$ mutation suggests that additional eIF $2 \alpha$ kinases partially complement the requirement for PERK in $\beta$ cell function (36).

Glucose not only promotes the secretion of insulin but also stimulates insulin transcription and translation (64-66). Our group has proposed that glucosestimulated proinsulin mRNA translation is regulated by PERK-mediated phosphorylation of eIF $2 \alpha$ in 
response to UPR activation 36). As blood glucose declines, energy may become limiting for protein folding in the ER and therefore activate the UPR to promote PERK-mediated phosphorylation of eIF $2 \alpha$. Conversely, a rise in blood glucose would turn off the UPR so that translation would accelerate, allowing entry of new preproinsulin into the ER. In this manner, PERKmediated phosphorylation of eIF $2 \alpha$ provides a brake on protein synthesis, including proinsulin translation. Continual elevation of blood glucose may also prolong elevated proinsulin translation, eventually activating the UPR as the secretion capacity of the ER is overwhelmed. Therefore, a delicate balance between glucose levels and eIF2 $\alpha$ phosphorylation needs to be maintained: Disturbances in either direction may lead to excessive UPR activation, with eventual $\beta$ cell death.

The insulin resistance and hyperglycemia associated with type 2 diabetes is accommodated by an increase in proinsulin translation. Under these conditions the UPR is activated to compensate for the increased protein-folding requirement in the ER. Prolonged activation of the UPR could contribute to the $\beta$ cell death associated with insulin resistance. Thus, the signaling mechanisms that $\beta$ cells use for sensing glucose levels, triggering insulin secretion, and rapidly controlling insulin biosynthesis may have coevolved with ER signaling pathways to support these specialized functions. Pancreatic $\beta$ cells are exquisitely sensitive to physiological fluctuations in blood glucose, because, in contrast to other cell types, they lack hexokinase, an enzyme with a low affinity but a high capacity for binding glucose. Therefore, in $\beta$ cells, the production of glucose 6-phosphate and the production of ATP through glycolysis are controlled by glucokinase (67), and the ratio of ATP to ADP correlates directly with the blood glucose level. Periodic decreases in blood glucose level (as occurs between meals) would decrease the $\mathrm{ATP} / \mathrm{ADP}$ ratio and compromise protein folding in the ER so that the UPR may be frequently activated in these cells. Hence, when glucose levels vary within the normal physiological range, the ER compartment of the $\beta$ cell may be exposed to greater energy fluctuations than is the ER of other cell types, making the $\beta$ cell uniquely dependent on the UPR for survival during intermittent decreases in blood glucose levels, as happens between meals. Additionally, the high-level expression of PERK and IRE1 $\alpha$ in the pancreas may predispose these kinases to dimerization and activation in response to intermittent stress.

\section{The UPR in organelle expansion}

The UPR is required for ER expansion that occurs upon differentiation of highly specialized secretory cells, but ER membrane expansion can also proceed independently of UPR activation. Overexpression of membrane proteins, such as HMG CoA reductase or the peroxisomal protein Pex15, promotes the expansion of smooth membranes without UPR activation $(68,69)$, as does overexpression of the $\mathrm{p} 180$ ribosome acceptor in the rough ER membrane (70). Conversely, protein overexpression, even under circumstances in which secretory capacity is unchanged (as occurs following the induction of high levels of cytochrome p450), can activate the UPR to induce ER chaperone levels to match the expanded membrane area $(71,72)$.

During the terminal differentiation of certain secretory cells, such as those in the pancreas or liver, membrane expansion is accompanied by a dramatic increase in protein secretion. Likewise, upon B cell maturation into high-level antibody-secreting plasma cells, the ER compartment expands approximately fivefold to accommodate the large increase in Ig synthesis. The requirement for the UPR in this latter process has been demonstrated in $\mathrm{XBP1}^{-/-}$cells. Since deletion of XBP1 produces an embryonic-lethal phenotype at day E14.5, the role of $X B P 1$ in $\mathrm{B}$ and $\mathrm{T}$ cell development had to be studied in immunoincompetent $R A G 1^{-/-}$mice reconstituted with $X B P 1^{-/}$embryonic stem cells (73). Work in these chimeric mice demonstrated that $X B P 1$ is required for high-level Ig production. Interestingly, the induction of Ig heavy-chain and light-chain gene rearrangement and the assembly and transport of Ig $\mu$ to the surface of the B cells occurred normally. However, plasma cells were not detected, suggesting a role for $X B P 1$ in plasma cell differentiation or survival.

These findings support the hypothesis that induction of Ig synthesis activates the UPR to induce ER expansion to accommodate the high-level antibody expression. Alternatively, activation of the UPR may be part of the differentiation program that occurs prior to induction of high-level antibody synthesis. Plasma cell differentiation is stimulated in vivo by treatment with LPS or by ligation of CD40 receptors, treatments that activate the innate immune response and have been shown to induce XBP1 mRNA splicing (19). Thus, the UPR may contribute to a programmed response to signals that increase a cell's protein-secretory demand.

\section{The UPR in hyperhomocysteinemia.}

The association between high levels of serum homocysteine and the development of ischemic heart disease and stroke is supported by substantial epidemiological data. Unfortunately, it is not known whether homocysteine is the underlying cause of atherosclerosis and thrombosis. Severe hyperhomocysteinemia is caused by mutation in the cystathionine $\beta$-synthase (CBS) gene, whose product is a vitamin B6-dependent enzyme required for the conversion of homocysteine to cysteine. Elevated homocysteine is also associated with vitamin B deficiency. In cultured vascular endothelial cells, homocysteine induces protein misfolding in the ER by interfering with disulfide bond formation, and it activates the UPR to induce expression of several ER stress response proteins, such as BiP, GRP94, CHOP, and HERP (74-76). Homocysteine also activates apoptosis in a manner that requires an intact IRE1-signaling pathway (76).

These findings suggest that homocysteine acts intracellularly to disrupt ER homoeostasis. Indeed, recent studies confirm that induction of hyperhomocysteinemia elicits UPR activation in the livers of normal or $\mathrm{Cbs}^{+-}$ mice (77). In addition, hyperhomocysteinemia activates 
SREBP cleavage, leading to intracellular accumulation of cholesterol (77). Increased cholesterol biosynthesis may explain the hepatic steatosis and possibly the atherosclerotic lesions associated with hyperhomocysteinemia. Finally, hyperhomocysteinemia accelerates atherosclerosis in $A \mathrm{poE}^{-/-}$mice $(78,79)$, although the molecular mechanisms remain to be elucidated.

Hyperhomocysteinemia is also associated with increased amyloid production and increased amyloid-mediated neuronal death in animal models of Alzheimer disease (80). These observations suggest that the UPR may link the disease etiologies of hyperhomocysteinemia and Alzheimer disease. HERP, a homocysteine-induced ER stress-responsive gene, appears to be involved in amyloid $\beta$-protein $(A \beta)$ accumulation, including the formation of senile plaques and vascular $A \beta$ deposits (81), and that it interacts with both presenilin-1 (PS1) and presenilin-2 (PS2), thus regulating presenilin-mediated $A \beta$ generation. Immunohistochemical analysis of brains from patients with Alzheimer disease reveals intense HERP staining in activated microglia in senile plaques.

\section{The UPR in cancer}

Hypoxia is a common feature of solid tumors that display increased malignancy, resistance to therapy, and poor prognosis. Hypoxia in the tumor results from increased demand due to dysregulated cell growth and from vascular abnormalities associated with cancerous tissue. The importance of hypoxia has been seen in the clinic, since it predicts for poor outcome of treatments, independent of treatment modality. Hypoxia activates the UPR, whose downstream signaling events can undermine the efficacy of treatment. Tumor cells need to adapt to the increasingly hypoxic environment that surrounds them as they grow, and the induction of the UPR is key to this response. Induction of the ER stress response genes, for example BiP and GRP94, in cancerous tissue correlates with malignancy, consistent with their antiapoptotic function (82). In addition, the UPR confers resistance to topoisomerase inhibitors, such as etoposide, and some UPR-induced genes directly mediate drug resistance via the multi-drug-resistance gene $M D R$. Therefore, approaches to prevent UPR activation in cancerous cells may significantly improve treatment outcome.

The proteasome inhibitor PS-341 is now in earlyphase clinical evaluation for the treatment of multiple myeloma, a clonal B cell tumor of differentiated plasma cells (83). The mechanism of PS-341 function is thought to be inhibition of IкB degradation, which prevents activation of the antiapoptotic transcription factor NF-кB. However, proteasomal inhibition would also prevent ERAD. As high-level heavy- or light-chain Ig production is likely associated with a certain degree of protein misfolding, it is possible that inhibition of ERAD function may be selectively toxic to B cell myelomas through activation of the UPR and apoptosis.

\section{The UPR and viral pathogenesis}

The two major mediators of the IFN-induced arm of the innate immune response are evolutionarily related to IRE1 and PERK. The kinase/endoribonuclease domain of IRE1 is homologous to RNaseL, and the protein kinase domain of PERK is related to the double-stranded RNA-activated (dsRNA-activated) eIF2 $\alpha$ protein kinase PKR. RNaseL and PKR mediate the IFNinduced antiviral response of the host, which is required to limit viral protein synthesis and pathogenesis. As part of the innate immune response to viral infection, RNaseL and PKR are activated by dsRNAs produced as intermediates in viral replication. In contrast to activation by dsRNA, IRE1 and PERK are activated by ER stress, which can be induced by high-level viral glycoprotein expression. All enveloped viruses produce excess glycoproteins that could elicit PERK and IRE1 activation to meet the need for increased folding and secretory capacity. More studies will be required to elucidate the role of the UPR in various viral diseases.

Hepatitis $\mathrm{C}$ virus $(\mathrm{HCV})$ is a positive-stranded RNA virus encoding a single polyprotein. Polyprotein cleavage generates at least ten polypeptides, including two glycoproteins, E1 and E2. A large amount of E1 forms disulfide-cross-linked aggregates with E2 in the ER (84). Since the accumulation of misfolded $\alpha 1$-PI elicits UPR activation, with subsequent hepatocyte death and hepatocellular carcinoma, it is possible that the aggregated E1/E2 complexes in the HCV-infected hepatocyte also contribute to hepatitis and hepatocellular carcinoma. Future studies should identify whether these glycoprotein aggregates activate the UPR to mediate the hepatocyte cell death and transformation associated with the pathogenesis of HCV infection.

\section{The UPR in tissue ischemia}

Finally, neuronal death due to reperfusion after ischemic injury is associated with activation of the UPR $(85,86)$. Immediately after reperfusion, protein synthesis is inhibited, due at least in part to phosphorylation of eIF $2 \alpha$; this inhibition may represent a protective mechanism to prevent further neuron damage. Recent studies support the idea that eIF2 $\alpha$ phosphorylation in response to reperfusion injury is mediated by PERK and hence that it depends on the UPR (87). If so, UPR activation prior to ischemic injury might protect the brain and other tissues from cell death during periods of reperfusion.

\section{Summary}

A variety of approaches have been employed to identify the UPR signaling components, their function, and their physiological role. Yeast genetics allowed the definition of the basic ER stress-signaling pathway. The identification of homologous and parallel signaling pathways in higher eukaryotes has produced a mechanistic framework the cell uses to sense and compensate for ER over-load and stress. The high-level tissue-specific expression patterns of several ER stress-signaling molecules indicated the pancreas and intestine as organs that require UPR for physiological function. Analysis of UPR-induced gene expression established that protein degradation is required to reduce the stress of unfolded protein accumulation in the ER. Major advances in identifying UPR function and rele- 
vance to disease were derived from mutation of UPR signaling components in model organisms and the identification of mutations in humans.

Despite tremendous progress, our knowledge of the UPR pathway remains incomplete. Further studies promise to expand our understanding of how ER stress impacts the other cellular signaling pathways. It will be very exciting and informative to understand how the UPR varies when critical components are genetically manipulated by deletion or other types of mutations. In addition, although the accumulation of unfolded protein in the ER is now known to contribute to pathogenesis in a variety of diseases, there are still few therapeutic approaches that target these events. With a greater understanding of protein-folding processes, pharmacological intervention with chemical chaperones to promote proper folding becomes feasible, as observed with sodium phenylbutyrate for $\Delta 508$ CFTR (see Gelman and Kopito, this Perspective series, ref. 53). Future intervention should consider activation of different subpathways of the UPR or overexpression of appropriate protein chaperones, as in the case of overexpression of the $\mathrm{J}$ domain of cytosolic HSP70, which suppresses polyglutamine toxicity in flies (88). Treatments that activate the ERAD response may also ameliorate pathogenesis in a number of the conformational diseases.

Over the past ten years, tremendous progress has been made in understanding the mechanisms and physiological significance of the UPR. The processes of protein folding and secretion, transcriptional and translational activation, and protein degradation are intimately interconnected to maintain homeostasis in the ER. A variety of environmental insults, genetic disease, and underlying genetic modifiers of UPR function contribute to the pathogenesis of different disease states. As we gain a greater understanding of the mechanisms that control UPR activation, it should be possible to discover methods to activate or inhibit the UPR as desired for therapeutic benefit.

\section{Acknowledgments}

I gratefully thank C. Yin-Liu, K. Zhang, E. McEwen, M. Cunningham, and D. Scheuner for comments on this manuscript and D. Scheuner for providing results prior to publication. This work was supported in part by NIH grant AI-42394 (to R.J. Kaufman).

1. Horwich, A. 2002. Protein aggregation in disease: a role for folding intermediates forming specific multimeric interactions. J. Clin. Invest. 110:1221-1232. doi:10.1172/JCI200216781.

2. Ron, D. 2002. Translational control in the endoplasmic reticulum stress response. J. Clin. Invest. 110:1383-1388. doi:10.1172/JCI200216784.

3. Lee, A.S. 1992. Mammalian stress response: induction of the glucose-regulated protein family. Curr. Opin. Cell Biol. 4:267-273.

4. Kaufman, R.J., et al. 2002. The unfolded protein response in nutrient sensing and differentiation. Nat. Rev. Mol. Cell Biol. 3:411-421.

5. Ellgaard, L., and Helenius, A. 2001. ER quality control: towards an understanding at the molecular level. Curr. Opin. Cell Biol. 13:431-437.

6. Jakob, C.A., Burda, P., Te Heesen, S., Aebi, M., and Roth, J. 1998. Genetic tailoring of $\mathrm{N}$-linked oligosaccharides: the role of glucose residues in glycoprotein processing of Saccharomyces cerevisiae in vivo. Glycobiology. 8:155-164.

7. Travers, K.J., et al. 2000. Functional and genomic analyses reveal an essential coordination between the unfolded protein response and ER-associated degradation. Cell. 101:249-258.

8. Kaufman, R.J. 1999. Stress signaling from the lumen of the endoplasmic reticulum: coordination of gene transcriptional and translational controls. Genes Dev. 13:1211-1233.

9. Mori, K. 2000. Tripartite management of unfolded proteins in the endoplasmic reticulum. Cell. 101:451-454.

10. Patil, C., and Walter, P. 2001. Intracellular signaling from the endoplasmic reticulum to the nucleus: the unfolded protein response in yeast and mammals. Curr. Opin. Cell Biol. 13:349-355.

11. Mori, K., Ogawa, N., Kawahara, T., Yanagi, H., and Yura, T. 2000. mRNA splicing-mediated C-terminal replacement of transcription factor Hac1p is required for efficient activation of the unfolded protein response. Proc. Natl. Acad. Sci. USA. 97:4660-4665.

12. Tirasophon, W., Welihinda, A.A., and Kaufman, R.J. 1998. A stress response pathway from the endoplasmic reticulum to the nucleus requires a novel bifunctional protein kinase/endoribonuclease (Ire1p) in mammalian cells. Genes Dev. 12:1812-1824.

13. Wang, X.Z., et al. 1998. Cloning of mammalian Ire1 reveals diversity in the ER stress responses. EMBO J. 17:5708-5717.

14. Niwa, M., Sidrauski, C., Kaufman, R.J., and Walter, P. 1999. A role for presenilin-1 in nuclear accumulation of Ire 1 fragments and induction of the mammalian unfolded protein response. Cell. 99:691-702.

15. Tirasophon, W., Lee, K., Callaghan, B., Welihinda, A., and Kaufman, R.J. 2000. The endoribonuclease activity of mammalian IRE1 autoregulates its mRNA and is required for the unfolded protein response. Genes Dev. 14:2725-2736.

16. Yoshida, H., Haze, K., Yanagi, H., Yura, T., and Mori, K. 1998. Identification of the cis-acting endoplasmic reticulum stress response element responsible for transcriptional induction of mammalian glucose-regulated proteins. Involvement of basic leucine zipper transcription factors. J. Biol. Chem. 273:33741-33749.

17. Yoshida, H., Matsui, T., Yamamoto, A., Okada, T., and Mori, K. 2001. XBP1 mRNA is induced by ATF6 and spliced by IRE1 in response to ER stress to produce a highly active transcription factor. Cell. 107:881-891.

18. Shen, X., et al. 2001. Complementary signaling pathways regulate the unfolded protein response and are required for C. elegans development. Cell. 107:893-903.

19. Calfon, M., et al. 2002. IRE1 couples endoplasmic reticulum load to secretory capacity by processing the XBP-1 mRNA. Nature. 415:92-96.

20. Lee, K., et al. 2002. IRE1-mediated unconventional mRNA splicing and $\mathrm{S} 2 \mathrm{P}-$ mediated ATF6 cleavage merge to regulate XBP1 in signaling the unfolded protein response. Genes Dev. 16:452-466.

21. Wang, Y., et al. 2000. Activation of ATF6 and an ATF6 DNA binding site by the endoplasmic reticulum stress response. J. Biol. Chem. 275:27013-27020.

22. Urano, F., et al. 2000. Coupling of stress in the ER to activation of JNK protein kinases by transmembrane protein kinase IRE1. Science. 287:664-666

23. Urano, F., Bertolotti, A., and Ron, D. 2000. IRE1 and efferent signaling from the endoplasmic reticulum. J. Cell Sci. 113:3697-3702.

24. Masaki, T., Yoshida, M., and Noguchi, S. 1999. Targeted disruption of CRE-binding factor TREB5 gene leads to cellular necrosis in cardiac myocytes at the embryonic stage. Biochem. Biophys. Res. Commun. 261:350-356.

25. Reimold, A.M., et al. 2000. An essential role in liver development for transcription factor XBP-1. Genes Dev. 14:152-157.

26 . Bertolotti, A., et al. 2001. Increased sensitivity to dextran sodium sulfate colitis in IRE1 $\beta$-deficient mice. J. Clin. Invest. 107:585-593.

27. Haze, K., Yoshida, H., Yanagi, H., Yura, T., and Mori, K. 1999. Mammalian transcription factor ATF6 is synthesized as a transmembrane protein and activated by proteolysis in response to endoplasmic reticulum stress. Mol. Biol. Cell. 10:3787-3799.

28. Yoshida, H., et al. 2000. ATF6 activated by proteolysis binds in the presence of NF-Y (CBF) directly to the cis-acting element responsible for the mammalian unfolded protein response. Mol. Cell. Biol. 20:6755-6767.

29. Li, M., et al. 2000. ATF6 as a transcription activator of the endoplasmic reticulum stress element: thapsigargin stress-induced changes and synergistic interactions with NF-Y and YY1. Mol. Cell. Biol. 20:5096-5106.

30. Yoshida, H., et al. 2001. Endoplasmic reticulum stress-induced formation of transcription factor complex ERSF including NF-Y (CBF) and activating transcription factors 6alpha and 6beta that activates the mammalian unfolded protein response. Mol. Cell. Biol. 21:1239-1248.

31. Ye, J., et al. 2000. ER stress induces cleavage of membrane-bound ATF6 by the same proteases that process SREBPs. Mol. Cell. 6:1355-1364.

32. Shen, J., Chen, X., Hendershot, L., and Prywes, R. 2002. ER stress regulation of ATF6 localization by dissociation of BiP/GRP78 binding and unmasking of Golgi localization signals. Dev. Cell. 3:99-111.

33. Nohturfft, A., Yabe, D., Goldstein, J.L., Brown, M.S., and Espenshade, P.J. 2000. Regulated step in cholesterol feedback localized to budding of SCAP from ER membranes. Cell. 102:315-323.

34. Clauss, I.M., Chu, M., Zhao, J.L., and Glimcher, L.H. 1996. The basic domain/leucine zipper protein hXBP-1 preferentially binds to and transactivates CRE-like sequences containing an ACGT core. Nucleic Acids Res. 24:1855-1864. 
35. Kokame, K., Kato, H., and Miyata, T. 2000. Identification of ERSE-II, a new cis-acting element responsible for the ATF6-dependent mammalian unfolded protein response. J. Biol. Chem. 275:32846-32853.

36. Scheuner, D., et al. 2001. Translational control is required for the unfolded protein response and in vivo glucose homeostasis. Mol. Cell. 7:1165-1176.

37. Harding, H.P., et al. 2000. Regulated translation initiation controls stressinduced gene expression in mammalian cells. Mol. Cell. 6:1099-1108.

38. Liu, C.Y., Schroder, M., and Kaufman, R.J. 2000. Ligand-independent dimerization activates the stress response kinases IRE1 and PERK in the lumen of the endoplasmic reticulum. J. Biol. Chem. 275:24881-24885.

39. Liu, C.Y., Wong, H.N., Schauerte, J.A., and Kaufman, R.J. 2002. The protein kinase/endoribonuclease IRE1a that signals the unfolded protein response has a lumenal amino-terminal ligand-independent dimerization domain. J. Biol. Chem. 277:18346-18356

40. Yoneda, T., et al. 2001. Activation of caspase-12, an endoplastic reticulum (ER) resident caspase, through tumor necrosis factor receptor-associated factor 2- dependent mechanism in response to the ER stress. J. Biol. Chem. 276:13935-13940.

41. Nishitoh, H., et al. 2002. ASK1 is essential for endoplasmic reticulum stress-induced neuronal cell death triggered by expanded polyglutamine repeats. Genes Dev. 16:1345-1355.

42. Leppa, S., and Bohmann, D. 1999. Diverse functions of JNK signaling and c-Jun in stress response and apoptosis. Oncogene. 18:6158-6162.

43. Ito, Y., et al. 2001. Targeting of the c-Abl tyrosine kinase to mitochondria in endoplasmic reticulum stress-induced apoptosis. Mol. Cell. Biol. 21:6233-6242.

44. Pinton, $\mathrm{P}$., et al. 2001. The $\mathrm{Ca} 2+$ concentration of the endoplasmic reticulum is a key determinant of ceramide-induced apoptosis: significance for the molecular mechanism of Bcl-2 action. EMBO J. 20:2690-2701.

45. Rao, R.V., et al. 2002. Coupling endoplasmic reticulum stress to the cell death program. An Apaf-1-independent intrinsic pathway. J. Biol. Chem. 277:21836-21842.

46. Nakagawa, T., et al. 2000. Caspase-12 mediates endoplasmic-reticulumspecific apoptosis and cytotoxicity by amyloid-beta. Nature. 403:98-103.

47. Morishima, N., Nakanishi, K., Takenouchi, H., Shibata, T., and Yasuhiko, Y. 2002. An ER stress-specific caspase cascade in apoptosis: cytochrome c-independent activation of caspase- 9 by caspase-12. J. Biol. Chem. In press.

48. Nakagawa, T., and Yuan, J. 2000. Cross-talk between two cysteine protease families. Activation of caspase-12 by calpain in apoptosis. J. Cell Biol. 150:887-804

49. Rao, R.V., et al. 2001. Coupling endoplasmic reticulum stress to the cell death program. Mechanism of caspase activation. J. Biol. Chem. 276:33869-33874.

50. Oyadomari, S., et al. 2002. Targeted disruption of the Chop gene delays endoplasmic reticulum stress-mediated diabetes. J. Clin. Invest. 109:525-532. doi:10.1172/JCI200214550.

51. Lomas, D.A., and Mahadeva, R. 2002. $\alpha 1$-Antitrypsin polymerization and the serpinopathies: pathobiology and prospects for therapy. J. Clin. Invest. In press. doi:10.1172/JCI200216782.

52. Perlmutter, D.H. 2002. Liver injury in $\alpha 1$-antitrypsin deficiency: role of autophagy and mitochondrial injury in response to aggregated protein in the endoplasmic reticulum. J. Clin. Invest. In press. doi:10.1172/JCI200216787.

53. Wu, Y., et al. 1994. A lag in intracellular degradation of mutant alpha 1-antitrypsin correlates with the liver disease phenotype in homozygous PiZZ alpha 1-antitrypsin deficiency. Proc. Natl. Acad. Sci. USA. 91:9014-9018.

54. Gelman, M.S., and Kopito, R.R. 2002. Rescuing protein conformation: prospects for pharmacological therapy in cystic fibrosis. J. Clin. Invest. In press. doi:10.1172/JCI200216786.

55. Lamande, S.R., and Bateman, J.F. 1999. Procollagen folding and assembly: the role of endoplasmic reticulum enzymes and molecular chaperones. Semin. Cell Dev. Biol. 10:455-464.

56. Delepine, M., et al. 2000. EIF2AK3, encoding translation initiation factor 2-alpha kinase 3, is mutated in patients with Wolcott-Rallison syndrome. Nat. Genet. 25:406-409.

57. Zhang, P., et al. 2002. The PERK eukaryotic initiation factor 2 alpha kinase is required for the development of the skeletal system, postnatal growth, and the function and viability of the pancreas. Mol. Cell. Biol. 22:3864-3874.

58. Bence, N.F., Sampat, R.M., and Kopito, R.R. 2001. Impairment of the ubiquitin-proteasome system by protein aggregation. Science. 292:1552-1555.

59. Kitada, T., et al. 1998. Mutations in the parkin gene cause autosomal recessive juvenile parkinsonism. Nature. 392:605-608.

60. Imai, Y., Soda, M., and Takahashi, R. 2000. Parkin suppresses unfolded protein stress-induced cell death through its E3 ubiquitin-protein ligase activity. J. Biol. Chem. 275:35661-35664.

61. Imai, Y., et al. 2001. An unfolded putative transmembrane polypeptide, which can lead to endoplasmic reticulum stress, is a substrate of Parkin. Cell. 105:891-902.
62. Imai, Y., et al. 2002. CHIP is associated with Parkin, a gene responsible for familial Parkinson's disease, and enhances its ubiquitin ligase activity. Mol. Cell. 10:55-67.

63. Harding, H.P., et al. 2001. Diabetes mellitus and exocrine pancreatic dysfunction in perk-/- mice reveals a role for translational control in secretory cell survival. Mol. Cell. 7:1153-1163.

64. Permutt, M.A. 1974. Effect of glucose on initiation and elongation rates in isolated rat pancreatic islets. J. Biol. Chem. 249:2738-2742.

65. Itoh, N., and Okamoto, H. 1980. Translational control of proinsulin synthesis by glucose. Nature. 283:100-102.

66. Lang, J. 1999. Molecular mechanisms and regulation of insulin exocytosis as a paradigm of endocrine secretion. Eur. J. Biochem. 259:3-17.

67. Schuit, F., Moens, K., Heimberg, H., and Pipeleers, D. 1999. Cellular origin of hexokinase in pancreatic islets. J. Biol. Chem. 274:32803-32809.

68. Masuda, A., Kuwano, M., and Shimada, T. 1983. Ultrastructural changes during the enhancement of cellular 3-hydroxy-3-methyl-glutaryl-coenzyme A reductase in a Chinese hamster cell mutant resistant to compactin (ML 236B). Cell Struct. Funct. 8:309-312.

69. Elgersma, Y., et al. 1997. Overexpression of Pex15p, a phosphorylated peroxisomal integral membrane protein required for peroxisome assembly in S. cerevisiae, causes proliferation of the endoplasmic reticulum membrane. EMBO J. 16:7326-7341.

70. Hyde, M., Block-Alper, L., Felix, J., Webster, P., and Meyer, D.I. 2002. Induction of secretory pathway components in yeast is associated with increased stability of their mRNA. J. Cell Biol. 156:993-1001.

71. Menzel, R., Vogel, F., Kargel, E., and Schunck, W.H. 1997. Inducible membranes in yeast: relation to the unfolded-protein-response pathway. Yeast. 13:1211-1229.

72. Takewaka, T., Zimmer, T., Hirata, A., Ohta, A., and Takagi, M. 1999. Null mutation in IRE1 gene inhibits overproduction of microsomal cytochrome P450Alk1 (CYP 52A3) and proliferation of the endoplasmic reticulum in Saccharomyces cerevisiae. J. Biochem. (Tokyo). 125:507-514.

73. Reimold, A.M., et al. 2001. Plasma cell differentiation requires the transcription factor XBP-1. Nature. 412:300-307.

74. Outinen, P.A., et al. 1999. Homocysteine-induced endoplasmic reticulum stress and growth arrest leads to specific changes in gene expression in human vascular endothelial cells. Blood. 94:959-967.

75. Huang, R.F., Huang, S.M., Lin, B.S., Wei, J.S., and Liu, T.Z. 2001. Homocysteine thiolactone induces apoptotic DNA damage mediated by increased intracellular hydrogen peroxide and caspase 3 activation in HL60 cells. Life Sci. 68:2799-2811.

76. Zhang, C., et al. 2001. Homocysteine induces programmed cell death in human vascular endothelial cells through activation of the unfolded protein response. J. Biol. Chem. 276:35867-35874.

77. Werstuck, G.H., et al. 2001. Homocysteine-induced endoplasmic reticulum stress causes dysregulation of the cholesterol and triglyceride biosynthetic pathways. J. Clin. Invest. 107:1263-1273.

78. Hofmann, M.A., et al. 2001. Hyperhomocysteinemia enhances vascular inflammation and accelerates atherosclerosis in a murine model. J. Clin. Invest. 107:675-683.

79. Zhou, J., et al. 2001. Dietary supplementation with methionine and homocysteine promotes early atherosclerosis but not plaque rupture in ApoE-deficient mice. Arterioscler. Thromb. Vasc. Biol. 21:1470-1476.

80. Kruman, I.I., et al. 2002. Folic acid deficiency and homocysteine impair DNA repair in hippocampal neurons and sensitize them to amyloid toxicity in experimental models of Alzheimer's disease. J. Neurosci. 22:1752-1762.

81. Sai, X., et al. 2002. Endoplasmic reticulum stress-inducible protein, Herp, enhances presenilin-mediated generation of amyloid beta-protein. J. Biol. Chem. 277:12915-12920

82. Lee, A.S. 2001. The glucose-regulated proteins: stress induction and clinical applications. Trends Biochem. Sci. 26:504-510.

83. Adams, J. 2002. Preclinical and clinical evaluation of proteasome inhibitor PS-341 for the treatment of cancer. Curr. Opin. Chem. Biol. 6:493-500.

84. Choukhi, A., Ung, S., Wychowski, C., and Dubuisson, J. 1998. Involvement of endoplasmic reticulum chaperones in the folding of hepatitis $\mathrm{C}$ virus glycoproteins. J. Virol. 72:3851-3858.

85. Little, E., Tocco, G., Baudry, M., Lee, A.S., and Schreiber, S.S. 1996. Induction of glucose-regulated protein (glucose-regulated protein $78 / \mathrm{BiP}$ and glucose-regulated protein 94) and heat shock protein 70 transcripts in the immature rat brain following status epilepticus. Neuroscience. 75:209-219.

86. DeGracia, D.J., Kumar, R., Owen, C.R., Krause, G.S., and White, B.C. 2002. Molecular pathways of protein synthesis inhibition during brain reperfusion: implications for neuronal survival or death. J. Cereb. Blood Flow Metab. 22:127-141.

87. Kumar, R., et al. 2001. Brain ischemia and reperfusion activates the eukaryotic initiation factor 2alpha kinase, PERK. J. Neurochem. 77:1418-1421.

88. Bonini, N.M. 2002. Chaperoning brain degeneration. Proc. Natl. Acad. Sci. USA. In press. 